\title{
TRADUCCIÓN LITERARIA VS. TRADUCCIÓN JURÍDICA: CONVERGENCIAS Y DIVERGENCIAS
}

\author{
LUDMILA ILIEVA \\ Universidad de Sofía San Clemente de Ojrid, Bulgaria
}

\begin{abstract}
Resumen. El artículo aborda el tema de las coincidencias entre Literatura y Derecho como dos actividades que atañen directamente la sociedad con su fin de formar y educar a los ciudadanos defendiendo sus intereses por medio de la ley o a través de la sanción moral. Un tema importante y controvertido que no puede ignorarse en los estudios de la traducción es el concepto de equivalencia. La comparación de distintas traducciones de un mismo texto implica inevitablemente distintas teorías de la equivalencia aplicadas a la traducción literaria y legal. Con este fin el artículo comprende un estudio de caso de la traducción a varios idiomas de un fragmento literario de temática jurídica.
\end{abstract}

Palabras clave: lenguaje, literatura, ley, traducción, equivalencia

\section{INTRODUCCIÓN}

En los últimos decenios ha venido creciendo el interés de la ciencia mundial hacia las investigaciones interdisciplinarias y no cabe duda de que la traducción es el campo más favorable para los estudios comparativos en el ámbito de las humanidades. Ello supone abordar los distintos tipos de traducción y su relación con los textos originales en la óptica tanto de la teoría general como de las teorías particulares de la traducción. Aparentemente los dos tipos de traducción literaria y jurídica, objeto de este artículo, no deberían tener muchos puntos en común, ¿por qué entonces este tema y esta comparación? La respuesta nos obliga a aclarar el vínculo entre lenguaje y Derecho como dos pilares en que se basa la sociedad humana, entre Literatura y Derecho por la interconexión histórica entre literatura y justicia y, por ende, su reflejo en la relación traducción literariatraducción jurídica.

\section{LENGUAJE Y DERECHO}

El lenguaje es uno de los productos más importantes de la vida espiritual del pueblo. Ya a finales del s. XX y principios del XXI la lingüística muestra un claro compromiso social que se expresa en el interés por el Derecho lo que lleva 
irremediablemente al encuentro entre lingüistas y juristas. En palabras del gran jurista español Antonio Hernández Gil, para el Derecho el lenguaje no es sólo un modo de exteriorizarse sino un modo de ser. En la palabra está encarnada la norma que rige la conducta de los hombres y en ella la precisión y la claridad actúan como verdaderos valores morales y no sólo estéticos. De allí que la justeza de la expresión no es extraña a la justicia del resultado y el Derecho impone al lenguaje una severa disciplina (Pasqual Liaño, 1979: 11).

Es decir, la forma de manifestación del Derecho es el lenguaje que le sirve como instrumento mientras que el Derecho legitima al poder.

El primer y fundamental cruce entre los dos fenómenos - lenguaje y Derecho - es que en ambos casos se trata de un sistema de reglas. Tanto el lenguaje como el Derecho son sistemas normativos (Tashev, 2001: 74).

Los teóricos son unánimes en que la traductología es una disciplina auxiliar al Derecho (Mounin, 1979: 9-17; Kasirer, 2005; Pelage, 2005). La comparación entre traductología y Derecho es acertada, más concretamente la comparación entre el trabajo del traductor y el del juez - ambos se enfrentan a una opción: en cada traducción, que podría ser de mucha responsabilidad, el traductor tiene que elegir entre palabras, sinónimos, construcciones cuyo uso erróneo podría tergiversar el sentido y causar daño. De la misma manera, ante la necesidad de dictar una sentencia, el juez sabe que a pesar de que exista el texto legal, la decisión definitiva la ha de tomar él. La responsabilidad es tan grande que muchas veces el juez se ve obligado a dirigirse a lo que otros han hecho en situaciones similares, a la jurisprudencia (Chukwu, 1991: 562-563). Así la simbiosis entre traductología (teoría de la traducción) y ciencia del Derecho da el inicio de una disciplina joven y todavía no muy conocida que es la traductología jurídica cuyo objetivo es fijar las reglas metodológicas aplicables a la traducción jurídica, planteando, por un lado, cuestiones de carácter general referentes a la metodología de la traducción como tal, independientemente del tipo concreto de traducción.

\section{LITERATURA Y DERECHO}

La relación intrínseca entre lenguaje y Derecho revela los vínculos entre traducción literaria y traducción jurídica. El tema de la relación entre Literatura y Derecho ha sido ampliamente explotado desde ópticas distintas - la de los teóricos de la literatura y críticos literarios, los filósofos y hermeneutas, los comparatistas. La Literatura y el Derecho son dos actividades cuyos lazos se inscriben dentro de una larga tradición histórica y atañen directamente la sociedad con su fin de formar y educar a los ciudadanos defendiendo sus intereses por medio de la ley o a través de la sanción moral. Derecho y Literatura son dos campos que pertenecen a disciplinas distintas pero permiten hablar de enlace entre ellos o de penetración recíproca: el Derecho incursiona en todas las esferas de la vida, entre ellas la Literatura, esta, por su parte, se nutre a menudo de temas que le ofrece el Derecho, relativos a categorías humanas, morales y estéticas como la vida, la muerte, el crimen, etc. tratadas con los instrumentos del Derecho. 
En ambos casos - de la Literatura y del Derecho - la principal forma de expresión es la escrita tratándose siempre de interpretación de textos. La filosofía del Derecho hace la distinción entre interpretación lingüística e interpretación jurídica. Hay autores que consideran que la interpretación lingüística es intuitiva mientras que la jurídica, racional (Tashev, 2001). La primera permite captar el sentido, reconstruirlo llenando las lagunas. En el Derecho esto sería buscar la lógica y la razón de la ley, necesarias para su aplicación.

Sin embargo, no hay que exagerar las similitudes entre textos literarios y jurídicos, respectivamente traducción de textos así, ya que existe una diferencia considerable y referente al efecto de ambos tipos de textos: en la Literatura el lector sabe que no debería tomar al pie de la letra lo que el escritor dice sino leer entre las líneas, en el Derecho no ocurre lo mismo, los textos jurídicos contienen reglas de cumplimiento obligatorio y han de ser comprendidos unívocamente (Marí, 1998: 180). Ello no impide el uso de metáforas y fórmulas retóricas en el Derecho. Los juristas y los jueces en concreto deben conocer y seleccionar muy bien las palabras y las formas de expresión con el fin de que el Derecho sea comprensible para todos, lo que supone indudablemente una preparación literaria, véase el mismo término letrado que hoy designa a un jurista, un hombre de leyes, mientras que inicialmente designaba al que tenía acceso a las letras, sabía leer y escribir, e.d. era alfabetizado, se le consideraba instruido, sabio.

\section{LA TRADUCCIÓN LITERARIA Y LA TRADUCCIÓN JURÍDICA ANTE EL PROBLEMA DE LA EQUIVALENCIA}

El tema de la Literatura y del Derecho como objeto de traducción se plantea sobre todo ante el dilema de la traducibilidad - según algunos, todo es traducible, según otros, la traducción es una empresa imposible, opiniones polares que siembran la duda en la posibilidad de decir lo mismo con otras palabras, e.d., estamos ante el problema de la equivalencia.

De hecho, todos los teóricos de la traducción emplean de una u otra forma el concepto de equivalencia como base teórica para definir la actividad traductiva cuyo objetivo es precisamente lograr la equivalencia.

Contestar a la pregunta ¿qué representa la equivalencia? significa decir si la traducción es possible o no. Y como la práctica ha comprobado que sí lo es, el problema consiste en qué tipo de equivalencia ha de buscar el traductor y cómo definirla.

La traducción literaria supone trasladar de un idioma a otro no sólo la información sino también una experiencia estética o artística. El objetivo del traductor literario es encontrar medios para trasladar el mensaje de tal forma que se produzca en el lector el mismo efecto que el autor pretende causar con su texto original, lo que no es nada fácil conociendo las diferencias de idiosincrasia y de conocimientos de fondo de los lectores. Se trata de encontrar equivalentes funcionales, concepto formulado en 1969 por el científico búlgaro Alexander 
Ludskanov que engloba el problema fundamental de la traducción: ésta no se reduce a trasmitir un medio por otro sino a transmitir las funciones de los medios en el sistema del todo, es decir, transmitir la información que ellos llevan por medios que cumplen funciones idénticas en la otra lengua y, por lo tanto, llevan la misma información (Ludskanov, 1969, 99-116).

A lo largo de los años aparecen múltiples modelos de traducción, muchos de ellos con indudables méritos, hasta que en Alemania surgen y se desarrollan las llamadas teorías funcionales de la traducción, orientadas a los tipos de textos y a las funciones del texto - la teoría de los tipos de texto de Katharina Reiss y la teoría del skopos de K. Reiss y Hans J. Vermeer.

Se acepta que el texto original y el traducido pueden diferir considerablemente no sólo como formulación y reparto del contenido sino también como objetivos que determinan la manera de ordenar el contenido (Vermeer, 2000: 223).

En este sentido las teorías funcionales realizan un cambio radical en la teoría de la traducción enfocando la traducción como un proceso comunicativo pragmático en que el texto original y el texto traducido pueden tener diferentes objetivos y funciones. Su mérito consiste en romper la vieja idea de las reglas de equivalencia que siempre han de ser aplicadas observando el requisito de fidelidad al texto original pero sin tomar en cuenta para qué y cómo se va a utilizar el texto traducido.

Todo ello atañe directamente la traducción jurídica. Según Susan Šarčevič, en la traducción de textos jurídicos (por ejemplo, contratos), la estrategia dependerá del ordenamiento legal - el de partida o el de llegada, el original o el de la traducción - según el cual se interpretará el texto (Šarčevič, 1997).

El enfoque a la traducción será diferente cuando se trata de textos jurídicos con fines informativos o con fines de aplicación en el país de la lengua de la traducción. En el primer caso Peter Newmark recomienda una traducción literal o semántica mientras que en el segundo, un enfoque comunicativo orientado a la lengua de la traducción. Newmark es uno de los pocos lingüistas que reconocen que el estatus de la traducción jurídica es instrumental cuando se trata de definir su empleo en la práctica (ibid.: 19).

Tradicionalmente la traducción de textos jurídicos obedece al principio de fidelidad al texto de partida, se considera que su tarea es reproducir la forma y el contenido del texto de partida con máxima exactitud - en los textos jurídicos la traducción literal es considerada como regla de oro, algo que aún hoy comparten no pocos juristas. Emmanuel Didier, por ejemplo, afirma que la traducción de leyes y otros textos normativos exige una precisión absoluta mientras que las resoluciones judiciales se pueden traducir más libremente, o sea, el tipo de texto desempeña determinado papel al definir la estrategia traductiva en el ámbito del Derecho (Didier 1990: 280-285, citado en Šarčevič, 2000: 3).

Así surge el concepto de equivalencia jurídica que marca una pauta en la historia y la ciencia de la traducción poniendo fin al criterio tradicional de conservar la letra del original, optando por un enfoque dinámico. 
Sin embargo, la práctica, basada en el criterio pragmático, tomando en consideración el efecto legal del original y de la traducción, demuestra que el concepto de equivalencia jurídica no se puede aplicar incondicionalmente y en varios casos, principalmente en un contexto en que hay dos o más lenguas oficiales y dos o más legislaciones, el traductor elige otro enfoque.

Se podría concluir que debido a la gran variedad de textos jurídicos, el enfoque funcional parece muy necesario ya que no elude la cuestión de la equivalencia sino la plantea desde otra óptica, rechazando la posibilidad de que exista un concepto de equivalencia universalmente aplicable.

$\mathrm{Y}$ en eso vemos de nuevo surgir el problema del tan anhelado modelo de traducción que hasta el momento no se ha podido lograr en su totalidad ni en el ámbito de la traducción literaria ni en el de la jurídica.

\section{ESTUDIO DE CASO}

Me gustaría ilustrar estos planteamientos con un caso práctico: la comparación de 5 traducciones a diferentes idiomas de un brevísimo fragmento de un cuento de Thomas Bernhard, en que el análisis lingüístico y el jurídico se completan (Ilieva, 2014: 9).

Die Welt ist eine ganz und gar, durch und durch juristische, wie Sie viel leicht nicht wissen. Die Welt ist eine einzige ungeheuere Jurisprudenz. Die Welt ist ein Zuchthaus! (Bernhard (2003) Ist es eine Komödie? Ist es eine Tragödie?)

Svetat e iztsialo iuriditcheski, kakto mozhe bi ne znaete. Svetat e edno edinstveno ogromno pravorazdavane. Svetat e zatvor.

(Bernhard (2006) Komedia li e? Tragedia li e?, (traducción de Maria Popova))

Vi, mozhet bit, ne znaete, no mir tselikom i polnostiu, ves naskvoz pronizan iurisprudentziei. Mir - sploshnaia tchudovishtnaia iurisprudentsia. Mir - eto katorga.

(Bernhard (2001) Komedia?.. Ili tragedia?, (traducción de Andrey Gordasevich)

El mundo es un mundo completamente, profundamente jurídico, como Usted quizás no lo sabe. El mundo es una sola, grandiosa jurisprudencia.

(Bernhard (2010) ¿Es una comedia?, ¿Es una tragedia? (traducción de Raffaele De Giorgi)

El mundo entero no es más que una jurisprudencia. El mundo entero es un presidio.

(Bernhard (2017) ¿Es una comedia?, ¿Es una tragedia? (traducción de Miguel Sáenz) 
Svetat e iztsialo podchinen na pravoto. Vie mozhe i da ne go znaete. Svetat e edno edinstveno ogromno pravorazdavane. Svetat e edna tamnitsa.

(Bernhard (2003) Komedia li e tova? Ili tragedia? (traducción de la autora))

Cuatro de las traducciones fueron encontradas en la red, la segunda traducción búlgara es mía, hecha para el caso concreto. Algunas traducciones son incompletas.

¿Qué es lo que nos revela la comparación de estas cinco traducciones? En primer lugar, la proximidad engañosa de los términos juristische y Jurisprudenz (dos palabras que derivan del latín - iuridicus - 'referente al Derecho y a la administración de justicia'), lo que podría llevar a la idea de que basta con trasladar los términos a la lengua de la traducción como préstamos culturales. En determinado contexto, sobre todo un contexto especializado, esto podría ser correcto - cuando la traducción está destinada a especialistas, concretamente juristas, la transferencia mecánica de jurídico y jurisprudencia es admisible inclusive a las dos lenguas eslavas en que la palabra originaria (pravo) - tiene otra etimología. El problema es si dos palabras, presentadas como traducción una de la otra, son equivalentes. ¿Permite la coincidencia literal de los términos considerar que son idénticos también por su sentido? Inclusive cuando la palabra es la misma en ambas lenguas ¿podría ella significar cosas distintas en uno y en otro Derecho?

Cuando se trata de lectores que no son especialistas, es obvio que el enfoque ha de ser diferente: buscar el sentido, la lógica del texto y no la exactitud del término. Para un lector común y corriente cuya lengua es eslava, la expresión El mundo es jurídico quedaría incomprensible - jurídico puede ser un problema, jurídica puede ser la carrera, la facultad, la revista pero no un concepto abstracto y global como el mundo. Según el Diccionario de la Lengua Búlgara, el adjetivo jurídico significa relacionado con el Derecho y tiene varios sinónimos - praven, pravov, legalen, zakonov - que en las lenguas no eslavas no se dan. Así que la interpretación debería orientarse a la amplificación del campo semántico el mundo está relacionado con el Derecho, está vinculado, dependiente, subordinado al Derecho.

El término jurisprudencia, que en los países mediterráneos de tradición romana justinianea se refiere a resoluciones judiciales idénticas, e.d. a la práctica del tribunal, en los países anglosajones está más cerca de lo que en aquellos es la Filosofía del Derecho en el marco de la Teoría General del Derecho. Vemos un parcial desencuentro en el sentido de un mismo término que en condiciones históricas distintas ha cobrado una orientación distinta y de acuerdo al contexto esta diferencia puede aumentar.

Así, jurisprudencia, según el diccionario, representa: 1) Un conjunto de ciencias jurídicas; 2) Práctica judicial en el sentido de interpretación idéntica de una misma norma por los tribunales. 
Ambas traducciones búlgaras optan por traducir jurisprudencia como pravorazdavane (administración de justicia).

Administrar justicia: Aplicar la justicia, procesar, judicializar.

No cabe duda de que desde la óptica del especialista los dos términos no son reemplazables, no es lo mismo aplicar las leyes y ocuparse de Derecho en general. La traducción literaria, sin embargo, admite y aspira a una equivalencia funcional - el mundo está subordinado al Derecho, se ha reducido únicamente a las reglas, a la aplicación de las leyes, está petrificado en ellas, se ha convertido en una administración de justicia y nada más. En nombre de la sugestión artística del texto, un desfase similar del sentido no es relevante ya que el sentido y la intención del autor están conservados, en la medida en que podemos estar seguros de que ésta ha sido su intención. La traducción literaria en su esencia es subjetiva, de ella no derivan efectos jurídicos y eso explica por qué podemos permitirnos cierta libertad de interpretación. Esta traducción no tiene relevancia jurídica.

Por otro lado hay una diferencia fundamental entre la tarea del traductor literario y la del autor del original en que prevalece la natural afinidad a la lengua mientras que en la traducción prevalecen las relaciones semánticas, el contenido (Stanchev, 2018: 146-151).

Evidentemente no ha sido esta la vía que ha seguido la reflexión del traductor ruso que prefirió trasmitir la sugestión, conservando el término original pero extendiendo la interpretación a otra faceta del sentido de jurídico: el Derecho está en todas partes, todo obedece a lo que ha dicho el tribunal, por lo cual utiliza un verbo mucho más expresivo - pronizat, pronizivat- penetrar, atravesar, causar dolor, atravesar con un objeto agudo o con un arma de fuego, la connotación es claramente negativa. Otra vez afrontamos una interpretación subjetiva que en la traducción jurídica no sería posible, es prácticamente inadmisible. A diferencia de sus colegas, el traductor ruso ha reforzado la interrogante, la inseguridad, la vacilación mediante los puntos suspensivos en el título.

En la primera traducción al español de Raffaele De Giorgi, supuestamente mexicano (no hay seguridad de que él es el traductor), parece que no se dio el problema de la interpretación - no se le puede culpar de haber interpretado erróneamente la intención del autor ya que traslada los dos términos tal cual, sin ningún cambio, contando probablemente con la colaboración del lector.

En cambio, tanto Miguel Sáenz, el traductor español, como Andrey Gordasevich, el ruso, eligen una palabra mucho más matizada emocionalmente: traducen Zuchthaus - (cárcel, prisión) - como katorga, presidio, lo que comparte la segunda traducción búlgara. Para el Derecho prisión y presidio son dos penas distintas: presidio es un tipo de pena por delitos criminales y políticos graves que supone un trabajo físico pesado y ha desaparecido hace mucho del sistema penal; prisión o cárcel es el lugar en que se tiene a las personas cumpliendo una sentencia firme que condena al imputado a prisión, a privación de libertad, lo mismo se refiere a los procesados o investigados a quienes se ha aplicado la medida de 
prisión preventiva. En un texto jurídico especializado estas penas suponen consecuencias, efectos jurídico-penales distintos.

\section{CONCLUSIONES}

La comparación entre cinco traducciones de un texto literario que contiene términos jurídicos pero no es técnicamente especializado, permite concluir que en el lenguaje del Derecho no siempre términos de etimología igual y que suenan de forma igual tienen el mismo sentido. Lo que es admisible en la traducción literaria, no siempre lo es en la jurídica. La metodología del análisis comparativo permite formular conclusiones útiles para la práctica de la traducción en el sentido del tipo de equivalencia al cual debería aspirar el traductor siendo el enfoque funcional necesario y apropiado y rechazando la posibilidad de que exista un concepto de equivalencia universalmente aplicable.

Así un breve ejemplo nos da la idea de la problemática inagotable de la traducción en sus distintas formas.

\section{REFERENCIAS BIBLIOGRÁFICAS}

Chukwu, U. (1991) Plaidoyer pour la part de la jurisprudence en traductologie. Meta, 36 (4): 562-563.

Didier, E. (1990) Langues et langages du droit. Montréal: Wilson\&Lafleur.

Gémar, J. C. y Kasirer, N. (2005) Jurilinguistique: entre langues et droits. Jurilinguistics: Between Law and Language. Montréal: Thémis/Bruylant.

Ludskanov, A. (1998) Principat na funktsionalnite ekvivalenti - osnova na teoriata y praktikata na prevoda. En E. Georgiev (ed.) Izkustvoto na perevoda (pp. 99-116). Sofia: Narodna cultura.

Marí, E. (1998) Derecho y literatura: algo de lo que sí se puede hablar pero en voz baja. Doxa, 21 (2): 251-287.

Mounin, G. (1979) La linguistique comme science auxiliaire dans les disciplines juridiques. Meta, 24 (1): 9-17.

Pasqual Liaño, M. (1997) Las peculiaridades del lenguaje jurídico desde la perspectiva del jurista. En P. San Ginés Aguilar, E. Ortega Arjonilla (eds.) Introducción a la traducción jurídica y jurada (p. 11). Granada: Comares.

Pelage, J. (2005) La traductologie, science auxiliaire du droit. Babilonia, Revista Lusofona de Linguas, Culturas e Traduçao, 2-3 (pp. 31-41). Lisboa: Universidade Lusofona de Humanidades e Technologias.

Šarčevič, S. (1997) New Approach to a Legal Translation. London: The Hague.

Šarčevič, S. (2000) Legal Translation and Translation Theory: A Receiver-oriented Approach. Disponible en http://www.tradulex.com/Actes2000/sarcevic.pdf [Consultado el 6 de abril de 2019].

Stanchev, Hr. (2018) Chuzhdosta y srodiavaneto na ezitsite v prevoda. Nabliudenia varhu prevodnata teoria na Walter Benjamin. En N. Stalianova (ed.) Bulgarska rech, godina XXIV, kniga 1 (pp. 146-151). Sofia: Universitetsko izdatelstvo Sv. Kliment Ohridski.

Tashev, R. (2001) Teoria na tilkuvaneto. Sofia: Sibi.

Vermeer, H. (2000) Skopos and Commission in Translational Action. En L. Venuti (ed.) The Translation Studies Reader (pp. 221-232). London: Routledge. 


\title{
RECURSOS DE ANÁLISIS EMPÍRICO
}

Bernhard, T. (2003) Erzählungen Kurzprose. Wien: Suhrkamp Velag.

Bernhard, T. ([1993] 2017) Relatos (traductor Miguel Sáenz). Madrid: Alianza Editorial.

Bernard, T. (2006) Komedia li e? Tragedia li e?(traducción de Maria Popova). Disponible en https: //www.hulite.net/modules.php?name=News\&file=article\&sid=55702 [Consultado el 12 de junio de 2019].

Bernard, T. (2001) Komedia? Ili Tragedia? (traducción de Andrey Gordasevich). Disponible en http://lib.ru/INPROZ/BERNHARD/Komoedie.txt_with-big-pictures.html [Consultado el 15 de mayo de 2019].

La jurisprudencia ¿es una comedia?, ¿es una tragedia? (2010) (traducción de Raffaele De Giorgi) Disponible en http://literaturayDerecho.blogspot.com/2010/06/la-jurisprudencia-esuna-comedia-es-una.html [Consultado el 4 de junio de 2019].

\section{LITERARY TRANSLATION VS. LEGAL TRANSLATION: CONVERGENCES AND DIVERGENCIES}

\begin{abstract}
The article addresses the topic of the coincidence between literature and law as two activities that directly affect society with the aim of educating citizens defending their interests through the law or through moral sanctions. A significant and controversial issue that cannot be ignored in translation studies is the concept of equivalence. The comparison of different translations of the same text inevitably involves different equivalence theories applied to literary and legal translation. That is why the article includes a case study of translation of a literary fragment of legal subject matter into several languages.
\end{abstract}

Key words: language, literature, law, translation, equivalence

Ludmila Ilieva (Dr. Phil., Prof. a titular) trabaja actualmente en la Universidad de Sofia San Clemente de Ojrid, Bulgaria. Sus intereses académicos incluyen la teoría y la práctica de la traducción, traducción literaria y especializada (jurídica y judicial), civilización de España y América Latina. Correo electrónico: ludmila.ilieva@gmail.com 